\title{
Survival and detection of coliforms, Enterobacteriaceae, and gram-negative bacteria in Greek yogurt
}

\author{
C. J. Hervert, N. H. Martin, K. J. Boor, and M. Wiedmann ${ }^{1}$ \\ Milk Quality Improvement Program, Department of Food Science, Cornell University, Ithaca, NY 14853
}

\begin{abstract}
Despite the widespread use of coliforms as indicator bacteria, increasing evidence suggests that the Enterobacteriaceae (EB) and total gram-negative groups more accurately reflect the hygienic status of high-temperature, short-time pasteurized milk and processing environments. If introduced into milk as postpasteurization contamination, these bacteria may grow to high levels and produce a wide range of sensory-related defects. However, limited information is available on the use and survival of bacterial hygiene indicators in dairy products outside of pasteurized fluid milk and cheese. The goal of this study was to (1) provide information on the survival of a diverse set of bacterial hygiene indicators in the low pH environment of Greek yogurt, (2) compare traditional and alternative detection methods for their ability to detect bacterial hygiene indicators in Greek yogurt, and (3) offer insight into optimal hygiene indicator groups for use in low-pH fermented dairy products. To this end, we screened 64 bacterial isolates, representing 24 dairy-relevant genera, for survival and detection in Greek yogurt using 5 testing methods. Before testing, isolates were inoculated into plain, $0 \%$ fat Greek yogurt (pH 4.35 to 4.65), followed by a 12-h hold period at $4 \pm 1^{\circ} \mathrm{C}$. Yogurts were subsequently tested using Coliform Petrifilm (3M, St. Paul, MN) to detect coliforms; Enterobacteriaceae Petrifilm (3M), violet red bile glucose agar and the D-Count (bioMérieux, Marcy-l'Étoile, France) to detect EB; and crystal violet tetrazolium agar (CVTA) to detect total gram-negative bacteria. Overall, the non-EB gram-negative isolates showed significantly larger $\log$ reductions $12 \mathrm{~h}$ after inoculation into Greek yogurt (based on bacterial numbers recovered on CVTA) compared with the coliform and noncoliform EB isolates tested. The methods evaluated varied in their ability to detect different microbial hygiene indicators in Greek yogurt. Crystal violet tetrazolium agar detected the highest portion of coliforms,
\end{abstract}

Received June 2, 2016.

Accepted October 22, 2016.

${ }^{1}$ Corresponding author: mw16@cornell.edu whereas EB Petrifilm detected the highest portion of $\mathrm{EB}$, as well as highest portion of total gram-negative bacteria. Additionally, the D-Count method allowed for faster detection of $\mathrm{EB}$ in yogurt by generating results in approximately $13 \mathrm{~h}$ rather than the $24 \mathrm{~h}$ required when using EB Petrifilm and violet red bile glucose agar. Results from this study indicate that the coliform and EB groups encompass a broad range of dairy-relevant gram-negative bacteria with the ability to survive in Greek yogurt, supporting their use as microbial hygiene indicator groups in low-pH fermented dairy products.

Key words: coliform, Enterobacteriaceae, yogurt, survival

\section{INTRODUCTION}

Dairy processors worldwide test for different gramnegative bacterial hygiene indicators to assess the quality of their finished products, evaluate sanitation practices at the processing level, and detect instances of postpasteurization contamination (Schröder, 1984; Ranieri and Boor, 2009). This testing represents a valuable tool for the dairy industry, allowing processors to identify areas of improvement within their operations that can be targeted to increase the quality and consumer acceptance of finished products (Silva et al., 2010; Costa Dias et al., 2012; Cusato et al., 2013). The majority of published research on dairy hygiene indicators focuses on coliforms in fluid milk and cheese (Martin et al., 2012; Masiello et al., 2016; Trmčić et al., 2016); there is currently little information on the use and detection of bacterial hygiene indicators in low$\mathrm{pH}$ fermented dairy products, such as Greek yogurt. Despite a steady decline in fluid milk consumption in the United States since the 1970s, both the production and consumption of Greek yogurt has greatly expanded in recent decades (Desai et al., 2013; USDA-ERS, 2015). The production of Greek yogurt, similar to that of traditional Swiss-style yogurt, involves processing steps to standardize, pasteurize, homogenize, and ferment milk. However, Greek yogurt production differs from traditional Swiss-style production in the processing steps that occur after fermentation. Specifically, 
Greek yogurt undergoes straining or centrifugation to separate acid whey from the curd to concentrate solids in the finished product. Although contamination of Greek yogurt with gram-negative organisms is likely to occur after the fermentation step (e.g., whey separation steps and packaging), there is the possibility of contamination before fermentation as well (e.g., valves and pipes at the postpasteurization stage, fermentation tanks). Therefore, research on the survival and detection of bacterial hygiene indicators in Greek yogurt would greatly benefit the growing number of yogurt processors looking to better assess sanitation practice and identify practices and procedures that may lead to postpasteurization contamination and affect finished product quality. The objectives of this research were to (1) provide dairy processors with information on the survival of a diverse set of bacterial hygiene indicators in the low $\mathrm{pH}$ environment of Greek yogurt, (2) compare traditional and alternative detection methods for these organisms, and (3) offer insight into optimal hygiene indicator groups for use in low-pH fermented dairy products.

Coliform bacteria have a long history of use as microbial hygiene indicators in the US dairy industry, dating back to 1914 (US Treasury Department, 1914). Currently, the Pasteurized Milk Ordinance requires US dairy processors to test grade "A" dairy products for the presence of coliforms as a means to assess finished product quality and hygiene (US FDA, 2011). Despite this, a recent study showed that a wide range of dairyrelevant gram-negative bacteria, whose presence in finished products would indicate postpasteurization contamination or other hygiene issues, go undetected on coliform selective and differential media (Hervert et al., 2016). Alternative indicator groups suggested for use in the dairy industry include the Enterobacteriaceae (EB) family and total gram-negative bacteria (Hervert et al., 2016). The primary advantage of testing for these alternative indicator groups is that tests for $\mathrm{EB}$ and total gram-negative bacteria detect the traditional coliform group of bacteria, as well as all other noncoliform gram-negative groups that represent common postpasteurization contaminants (Hervert et al., 2016).

By definition, coliforms are aerobic or facultatively anaerobic, gram-negative, non-spore-forming rods capable of fermenting lactose resulting in gas and acid production within $48 \mathrm{~h}$ at either $32^{\circ} \mathrm{C}$ or $35^{\circ} \mathrm{C}$ (Feng et al., 2002; Davidson et al., 2004). Traditional coliform genera include Escherichia, Klebsiella, Citrobacter, and Enterobacter, although bacteria in over 20 gramnegative genera meet the phenotypic criteria of coliforms (Imhoff, 2005; Masiello et al., 2016). Although EB share many of the phenotypic traits that define coliforms, classification into the EB family is on a taxonomic basis (Imhoff, 2005). The differential capabilities of most traditional EB detection methods rely on the ability of EB bacteria to ferment glucose, resulting in gas, acid, or gas and acid production. The EB family encompasses the vast majority of coliform bacteria (Imhoff, 2005) and includes several dairy-associated genera typically lacking the ability to ferment lactose (e.g., Salmonella and Yersinia; Imhoff, 2005). However, previous studies indicate that gram-negative bacteria outside of the coliform and EB groups (e.g., Pseudomonas and Acinetobacter) dominate the gram-negative microflora of pasteurized milk and go undetected when using coliform and EB detection methods (Ranieri and Boor, 2009; Hervert et al., 2016), supporting the value of testing for total gram-negative bacteria as hygiene indicators for at least some dairy products. Traditional detection methods for the total gram-negative group commonly contain a gram-positive inhibitor (e.g., crystal violet) to select for all coliform, EB, and non-EB gram-negative bacteria (Frank and Yousef, 2004).

Perhaps the most critical ability of microbial hygiene indicators is their capability to survive in the food matrix to the point of selective and differential testing. For example, pasteurized milk, with its $\mathrm{pH}$ close to neutral (approximately 6.7), contains key nutrients that are ideal for sustaining growth and survival of microbial hygiene indicators over the course of product shelf life. Yogurt, while still possessing key nutrients required for microbial growth, typically ranges in $\mathrm{pH}$ between 4.2 and 4.6. The relatively low $\mathrm{pH}$ of yogurt and other fermented dairy products is a result of the fermentation of milk lactose into lactic acid through the activity of lactic acid bacteria usually added as starter cultures (Vedamuthu, 2007). Yogurt starter cultures contain Streptococcus salivarius ssp. thermophilus and Lactobacillus delbrueckii ssp. bulgaricus, which largely out-compete other bacteria present in milk due the inhibitory effect of the lactic acid, utilization of the primary carbohydrate source (lactose), as well as the production of other inhibitory compounds, such as hydrogen peroxide (Dave and Shah, 1997; Vedamuthu, 2007).

Although the growth of coliform, EB, and gramnegative bacteria in pasteurized fluid milk at refrigeration temperatures is well documented (Martin et al., 2012; Masiello et al., 2016), limited research exists on the survival of microbial hygiene indicators in low-pH fermented dairy products. Existing research, though lacking in the diversity of organisms evaluated, suggests a limited ability of coliforms and $\mathrm{EB}$ to survive in low-pH fermented dairy products (Goel et al., 1971; Shaker et al., 2008). This presents a unique issue to 
yogurt processors who use bacterial hygiene indicator tests to evaluate their finished products. Specifically, the inhibitory factors (e.g., low $\mathrm{pH}$, starter culture interaction) inherent to Greek yogurt may injure bacterial indicator organisms present in the finished product, leading to a reduction in indicator organism detection, particularly with selective and differential test methods. Therefore, data on the recovery of gram-negative bacterial hygiene indicators following their inoculation and hold in Greek yogurt would provide valuable insight into ideal detection methods for use in the dairy industry. In a recent study (Hervert et al., 2016), 211 dairy-relevant coliform, $\mathrm{EB}$, and gram-negative isolates were screened for detection on Coliform Petrifilm (3M, St. Paul, MN), EB Petrifilm (3M), violet red bile glucose agar (VRBGA), crystal violet tetrazolium agar (CVTA), and a rapid flow cytometry-based method. For the study reported here, we selected a 64-isolate subset from the 211 isolates used in the previous study (Hervert et al., 2016) to undergo inoculation into Greek yogurt, followed by testing using the indicator organism detection methods listed above. The resulting data provide new information on the ability of different tests to detect bacterial hygiene indicators in the presence of competitive microflora and inhibitory compounds found in yogurt. Importantly, the testing procedures used in this study mirror those performed in industry settings. Thus, our results are highly relevant to yogurt processors looking to maximize their detection of a wide range of dairy-relevant gram-negative bacteria whose detection in finished products may indicate the occurrence of postpasteurization contamination.

\section{MATERIALS AND METHODS}

\section{Isolate Selection}

Based on results from Hervert et al. (2016) and information listed in the Food Microbe Tracker Database (www.foodmicrobetracker.com; Vangay et al., 2013), a collection of 64 gram-negative bacterial isolates representing a broad range of dairy-relevant bacteria was assembled for the study reported here. The selected isolate set contained $54 \mathrm{~EB}$ isolates from 19 genera and 10 non-EB gram-negative isolates from 5 genera. Identification of isolates to a genus level was based on previously completed $16 \mathrm{~S}$ rDNA sequencing as described in prior studies (Huck et al., 2007). Among the 54 EB isolates selected, 42 were identified as coliform bacteria based on their ability to ferment lactose resulting in colonies with associated gas bubbles when plated on Coliform Petrifilm (Hervert et al., 2016). Additionally, isolates were selected based on growth characteristics observed when pure cultures were evaluated in a previ- ous study (Hervert et al., 2016), with preference given to isolates whose plate counts on selective and differential media corresponded well with those expected based on enumeration on brain-heart infusion (BHI) agar of cultures grown in BHI broth. Isolates were also selected to represent dairy-associated isolation sources, with all but one isolate coming from dairy products or processing environments. Two non-EB isolates (Brevundimonas isolates FSL C4-0016 and C4-0057) were included, despite the fact that previous data (Hervert et al., 2016) indicated no growth on any of the media tested here, because they represented a genus isolated from dairy products (see Supplemental Table S1; https:// doi.org/10.3168/jds.2016-11553) and because it is possible (albeit unlikely) that isolates may be detectable with a given method (particularly flow cytometry) after exposure to yogurt, even if they are not detectable after growth in BHI media.

\section{Inoculation and Subsequent Testing of Greek Yogurts}

Isolates were streaked onto BHI agar (Becton Dickinson, Sparks, MD) from frozen culture, followed by incubation at $32 \pm 1^{\circ} \mathrm{C}$ for $24 \mathrm{~h}$. Individual colonies were then used to inoculate $5 \mathrm{~mL}$ of BHI broth (Becton Dickinson), followed by $18 \mathrm{~h}$ of incubation (no aeration) at $32 \pm 1^{\circ} \mathrm{C}$. Based on previously generated growth data (Hervert et al., 2016), BHI broth cultures were serially diluted using sterile phosphate buffer to concentrations that facilitated inoculation, at target levels of approximately 300 to $2,000 \mathrm{cfu} / \mathrm{g}$, of $0 \%$ fat, plain Greek yogurt (actual inoculation levels enumerated on BHI are shown in Supplemental Table S1; https:// doi.org/10.3168/jds.2016-11553). Isolates that did not yield colonies on at least one of the media used for detection at the targeted yogurt inoculation levels were retested at inoculum concentrations $\geq 1 \log$ greater. The sealed yogurts were inoculated from the base of the cup using a sterile 18-gauge needle and syringe so that the headspace of the packaging was not altered. Immediately following inoculation, the hole from the needle was sealed using sterile, adhesive foil. Inoculated yogurts were then held for $12 \mathrm{~h}$ at $4 \pm 1^{\circ} \mathrm{C}$ before testing using the selective and differential detection methods. These methods included (1) Coliform Petrifilm (3M) for coliform detection; (2) EB Petrifilm (3M), VRBGA (Becton Dickinson), and a flow cytometry instrument for EB detection (D-Count; bioMérieux, Marcy-l'Étoile, France); and (3) CVTA (Frank and Yousef, 2004) for detection of total gram-negative bacteria. To plate on Coliform Petrifilm, EB Petrifilm, VRBGA, and CVTA, a 1:10 serial dilution of the yogurt was prepared with sterile phosphate buffer, and the $\mathrm{pH}$ of the resulting 
dilution was adjusted to between 6.6 and 7.2 using 1 $N \mathrm{NaOH}$; the maximum volume of $\mathrm{NaOH}$ added was $<1.5 \%$ of the total volume. A $1-\mathrm{mL}$ aliquot of each $\mathrm{pH}$-adjusted yogurt dilution was plated in duplicate on Coliform Petrifilm and EB Petrifilm, and $250-\mu \mathrm{L}$ aliquots were plated in duplicate on CVTA and VRBGA using the nonexponential mode of an automated spiral plater (Advanced Instruments Inc., Norwood, MA). Thus, Coliform and EB Petrifilms both had detection limits of $5 \mathrm{cfu} / \mathrm{g}$ of yogurt, whereas CVTA and VRBGA plates had detection limits of $20 \mathrm{cfu} / \mathrm{g}$ of yogurt. Coliform Petrifilms, EB Petrifilms, and VRBGA plates were incubated for $24 \mathrm{~h}$ at $32 \pm 1^{\circ} \mathrm{C}$ and CVTA plates were incubated for $48 \mathrm{~h}$ at $21 \pm 1^{\circ} \mathrm{C}$ before enumeration (Frank and Yousef, 2004). In addition to plating on traditional detection media, yogurts were tested in duplicate using a flow cytometry method (D-Count) with a protocol designed to detect EB bacteria (protocol "Presence/Absence Test of EB in Fermented Milk Products Containing Bifidobacterium"); this procedure includes an enrichment step, followed by flow cytometry to allow for EB detection in $13 \mathrm{~h}$ rather than 24 h. Briefly, for each isolate tested, $1 \mathrm{~g}$ of the inoculated yogurt was transferred into $9 \mathrm{~mL}$ of a proprietary $\mathrm{EB}$ selective broth. Inoculated broths were incubated for $13 \mathrm{~h}$ at $37^{\circ} \mathrm{C} \pm 1^{\circ} \mathrm{C}$ before testing on the D-Count. To perform the final test, $10 \mu \mathrm{L}$ of enrichment broth was treated with reagents that label viable EB cells. The sample was then automatically injected into the flow cell analyzer of the D-count and the detectors within the analyzer counted the labeled cells, outputting a value in counts per milliliter of analyzed sample. In addition to testing the inoculated yogurts using the 5 methods described above, an uninoculated yogurt from the same manufacturing lot used for the inoculated yogurts was tested, in duplicate, to serve as a plating and background contamination negative control. The testing procedures used here mirrored those performed by Hervert et al. (2016) to ensure consistency in interpretation results for isolates that were tested in both pure culture and Greek yogurt.

\section{Interpretation of Growth Results}

Test methods were interpreted as outlined by Hervert et al. (2016). Briefly, following the incubation of plates and Petrifilms, colony growth was enumerated visually for Petrifilms, and with the aid of a Color Q-Count (Advanced Instruments Inc.) instrument for plates. Differential media types were also examined for typical or atypical growth characteristics indicating a positive or negative result for a medium type's respective differential capabilities. For EB Petrifilm, a positive result was indicated by acid, gas, or gas and acid production generated through the fermentation of glucose. Per the manufacturer's instructions, these characteristics resulted in red colonies with yellow zones for acid-producing isolates, red colonies with associated gas bubbles for gas-producing isolates, and red colonies with yellow zones and associated gas bubbles for acid- and gas-producing isolates. Enterobacteriaceae glucose fermenters produced red colonies on VRBGA with red-purple halos (bile precipitation) indicating acidification identified by neutral red, a $\mathrm{pH}$ indicator. Lactose-fermenting isolates were classified as coliforms and were identified through formation of red colonies with associated gas bubbles when plated on Coliform Petrifilm, as outlined in the manufacturer's instructions. On CVTA, dark red colonies indicated characteristic growth of gram-negative isolates (Frank and Yousef, 2004). For the D-Count, a positive result for an EB organism was defined as an instrument reading indicating $\geq 100$ counts $/ \mathrm{mL}$.

\section{Data Analyses}

All data were managed using Excel (version 14.5.4, Microsoft Corp., Redmond, WA) and all statistical analyses were performed using RStudio (version 0.98.149, RStudio Inc., Boston, MA). Sensitivity, or the true positive rate, was defined as the proportion of true positives that were correctly identified as such. Specificity, or the true negative rate, was defined as the proportion of true negatives that were correctly identified as such. True-positive and true-negative test results were assessed based on the target organism group for a specific test (i.e., coliform, EB, and total gramnegative bacteria). For the purpose of this study, isolate detection refers to the growth of an isolate with typical characteristics when evaluated using a given testing method. On the other hand, atypical growth on CVTA (i.e., colonies without red color) was used in some cases (i.e., 9 isolates) to provide enumeration data to assess isolate survival. These same instances of isolates showing atypical growth on CVTA were counted as negative test results when evaluating the sensitivity of CVTA (as they presented atypical growth). An ANOVA and a Tukey's honest significant difference test were performed to compare the log differences between bacterial levels inoculated into Greek yogurt and those recovered on CVTA for coliforms, noncoliform EB, and non-EB gram-negative bacteria.

\section{RESULTS AND DISCUSSION}

A total of 64 gram-negative bacterial isolates were inoculated into Greek yogurt, followed by incubation at $4 \pm 1^{\circ} \mathrm{C}$ for $12 \mathrm{~h}$; inoculation levels were determined 
by enumeration of the inoculum on BHI. After incubation of the inoculated yogurt, bacterial numbers were enumerated using plate counts determined on Coliform Petrifilm, EB Petrifilm, VRBGA, and CVTA. The resulting data were used to assess bacterial survival in Greek yogurt over $12 \mathrm{~h}$ and the ability of different media to recover bacterial isolates that could be introduced into yogurt through postpasteurization contamination.

\section{Non-EB Gram-Negative Bacteria Tested in Greek Yogurt Exhibited Rapid Die-Off with Limited Recovery on Selective and Differential Media}

Bacterial enumeration data on CVTA, which selects for total gram-negative bacteria, were used to assess growth and survival of gram-negative bacteria in Greek yogurt at $12 \mathrm{~h}$ after inoculation. Among the 64 gramnegative isolates inoculated into Greek yogurt, no appropriate enumeration data to assess growth or survival were generated for 8 isolates. These 8 isolates included 3 isolates (Plesiomonas isolate FSL Y1-0254; Brevundimonas isolates FSL C4-0016 and C4-0057) known to not exhibit growth on CVTA, 2 isolates (Flavobacterium isolates FSL R5-0497 and R5-0610) that grew with atypical growth on CVTA (brown film-like growth that did not present as countable colonies), and 3 isolates (Pantoea isolate FSL P4-0767; Proteus isolates FSL A50110 and A5-0127) for which inoculation levels could not be determined due to spreading colony morphologies on BHI. The 56 isolates with enumeration data on CVTA could be grouped into 3 categories: no change in bacterial numbers $(<0.5 \log$ increase or decrease in numbers), moderate die-off (between 0.5 and $1.0 \mathrm{log}$ decrease in numbers), and $>1.0 \log$ die-off. Aeromonas isolates FSL C4-0005 and R5-0758 showed no countable colonies; these isolates grew with typical colony characteristics when tested in pure culture (Supplemental Table S1; https://doi.org/10.3168/jds.2016-11553), so this suggests that their numbers dropped below the detection limit during the 12-h hold in Greek yogurt. Reduction of these isolates was thus calculated, using the detection limit on CVTA, as $>3.08 \log$ and $>3.06$ $\log$ for FSL C4-0005 and R5-0758, respectively; these isolates were thus classified into the $>1 \log$ die-off category.

All 42 coliform isolates tested in this study showed growth on the total gram-negative medium, CVTA. Most of these isolates $(71 \%$; 30/42) showed no change in bacterial numbers over the 12-h hold period in Greek yogurt (Figure 1). The remaining coliform isolates showed either moderate die-off $(21 \% ; 9 / 42)$ or $>1 \log$ die-off $(7 \% ; 3 / 42)$. The 30 coliform isolates with no change in bacterial numbers represented 12 different genera, including 3 Hafnia and 3 Klebsiella isolates, which all showed numerical increases close to $0.5 \mathrm{log}$ (ranging from 0.38 to 0.47 log; Figure 1). Hafnia was previously identified as part of the microbial population of raw milk cheeses and can even represent the dominant bacterial population in some cheese varieties made from raw milk (Wolfe et al., 2014; Trmčić et al., 2016). Although these previous data support the ability of Hafnia to survive mild acidification, the ability of representatives of this genus to survive very low $\mathrm{pH}$ values typical for yogurt had not been previously reported, to our knowledge. Additionally, we did not identify other studies that reported the effective survival of Klebsiella in yogurt. The ability of Hafnia and Klebsiella to readily survive the low $\mathrm{pH}$ of Greek yogurt suggests that representatives of these genera, and specifically the isolates tested here, may be candidate organisms for challenge studies involving microbial hygiene indicators in low-pH dairy foods. Interestingly, all 3 Escherichia isolates tested also survived over the 12-h hold period. By comparison, a previous study reported a reduction of $E$. coli in inoculated yogurt stored at $4^{\circ} \mathrm{C}$ from 3.8 to $1.9 \log \mathrm{cfu} / \mathrm{g}$ for a nonpathogenic $E$. colis strain and from 4.4 to $3.6 \log \mathrm{cfu} / \mathrm{g}$ for a pathogenic E. coli strain over a 72-h hold period (Bachrouri et al., 2002); the nonpathogenic and pathogenic E. coli strain levels declined below detection limit $(10 \mathrm{cfu} / \mathrm{g})$ after 168 and $312 \mathrm{~h}$ of incubation, respectively (Bachrouri et al., 2002). The $<1 \log$ reduction seen for the pathogenic E. coli strain over $72 \mathrm{~h}$ further supports potentially limited die-off of at least some $E$. coli under the type of $\mathrm{pH}$ stress encountered in yogurt (Bachrouri et al., 2002).

Notably, 9 of the 12 coliform isolates showing moderate (between 0.5 and $1.0 \mathrm{log}$ ) or $>1.0 \log$ decreases in numbers were classified into the genera Buttiauxella, Cronobacter, or Rahnella, and the remaining 3 coliform isolates represented the genera Cedecea, Citrobacter, and Enterobacter. These results are consistent with previous studies (Goel et al., 1971; Shaker et al., 2008) evaluating the survival of typical coliform genera in fermented dairy products. For example, one study that assessed the survival of Enterobacter sakazakii (now Cronobacter) in yogurt suggests an approximately 2 $\log$ reduction in E. sakazakii populations over the 20 $\mathrm{h}$ after the yogurt reaches a $\mathrm{pH}$ of about 4.7 (Shaker et al., 2008). Another study found that Aerobacter (Enterobacter) aerogenes rapidly dies off following its inoculation into yogurt with approximately 1 to $>2 \log$ reductions over $24 \mathrm{~h}$ of refrigerated storage (Goel et al., 1971).

Of the 56 total isolates included in our analysis of survival in Greek yogurt, 8 represented noncoliform EB bacteria in the genera Pantoea, Proteus, Salmo- 
nella, and Yersinia. Within this set, 5 isolates showed no change in numbers over the hold period whereas 3 showed moderate decreases in numbers (Figure 1). Statistical analysis showed no significant difference between the $\log$ differences of the 42 coliform and the 8 noncoliform EB isolates tested $(P=0.81)$. Notably, the 3 isolates showing moderate die-off were classified as Salmonella. By comparison, a previous study evaluating the viability of Salmonella in yogurt prepared with probiotic starter cultures reported a slight numerical increase in Salmonella numbers over the first $24 \mathrm{~h}$ of storage at $4^{\circ} \mathrm{C}$ (Nassib et al., 2006); after the initial 24 h, Nassib et al. (2006) observed a $>1 \log$ reduction in viable Salmonella over the next $48 \mathrm{~h}$ of cold storage. On the other hand, the 3 Yersinia isolates tested in this study showed the ability to survive in Greek yogurt over $12 \mathrm{~h}$ with numerical increases ranging from 0.05 to $0.18 \mathrm{log}$. Consistent with these observations, a previous study evaluating the survival of Yersinia enterocolitica in yogurt held at $4^{\circ} \mathrm{C}$ reported a $0.5 \log$ increase in $Y$. enterocolitica numbers during the first $5 \mathrm{~d}$ of storage, with a decrease of $>2.5 \log$ over the remaining $21 \mathrm{~d}$ of the study (Aykut and Oezbas, 1994). Given its psychrotrophic nature and ability to survive in low pH Greek yogurt (Champagne et al., 1994), postpasteurization contamination of yogurts with $Y$. enterocolitica could thus be a concern (Tacket et al., 1984).

Finally, 6 of the 56 total isolates included in our survival analysis fell into 1 of 3 non-EB gram-negative genera (i.e., Acinetobacter, Aeromonas, and Pseudomonas). All 6 isolates of this group showed high die-off in Greek yogurt with $>1.0$ log decrease in numbers over the 12-h hold period (Figure 1). Overall, the non-EB gram-negative isolates showed significantly larger log reductions at $12 \mathrm{~h}$ after inoculation into Greek yogurt (based on bacterial numbers recovered on CVTA) compared with the coliform $(P<0.0001)$ and noncoliform EB isolates tested $(P<0.0001)$. These results suggest that non-EB gram-negative bacteria may be more susceptible to the low $\mathrm{pH}$ of yogurt than coliform and EB. The 2 Acinetobacter isolates (FSL C4-0013 and C4-0087) showed reductions of 1.01 and $1.95 \mathrm{log}$, respectively; we are not aware of other studies that have evaluated the survival of Acinetobacter in yogurt.
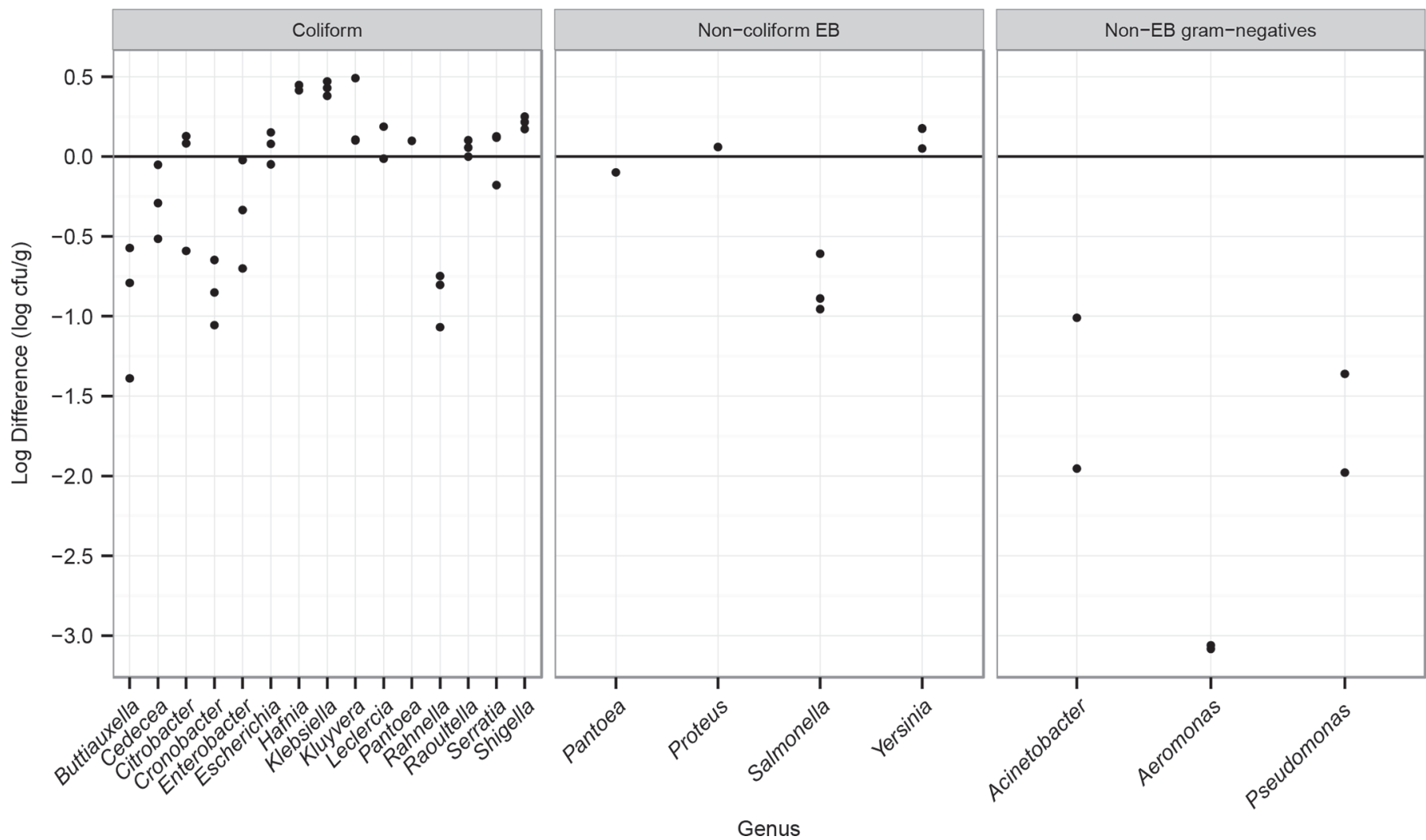

Figure 1. Recovery of 42 coliform and 8 noncoliform Enterobacteriaceae (EB) as well as 6 non-EB gram-negative isolates on crystal violet tetrazolium agar (CVTA). Plotted values represent the log difference between the count (cfu) inoculated into Greek yogurt and that recovered on CVTA per gram of yogurt. 
Neither of the Aeromonas isolates tested in this study exhibited recovery on CVTA even though the preliminary tests in pure culture showed that both isolates can be recovered on this medium (Hervert et al., 2016). This suggests that they did not survive the 12-h hold period; alternatively, cell injury could have prevented growth on CVTA during the 48-h incubation period. A previous study testing the survival of Aeromonas in yogurt saw similar results in that $A$. hydrophilia numbers decreased $>6 \log$ over the first $5 \mathrm{~d}$ of storage at $4^{\circ} \mathrm{C}$ (Aykut and Oezbas, 1994). The 2 Pseudomonas isolates tested in this study showed a $>1 \log$ reduction over the 12-h hold in the yogurt (1.36 and $1.98 \log$ for isolates FSL R5-0318 and W7-0098, respectively). Although limited research exists on the survival of Pseudomonas in yogurt, a previous study noted the ability of Pseudomonas paucimobilis to survive up to $45 \mathrm{~d}$ in yogurts inoculated at approximately $10^{8} \mathrm{cfu} / \mathrm{mL}$ (Canganella et al., 1999). However, Canganella et al. (1999) acknowledge that Pseudomonas paucimobilis is not a common contaminant of fermented milk and is more commonly associated with nondairy environments. Consistent with the yogurt survival results obtained here, a previous study reported $>1 \log$ reductions in viable cells for 3 Pseudomonas strains inoculated into Greek yogurt over the first $24 \mathrm{~h}$ of storage at $6^{\circ} \mathrm{C}$ (Birollo et al., 2001). Furthermore, the 3 Pseudomonas strains tested by Birollo et al. (2001) were undetectable after $84 \mathrm{~h}$ of refrigerated storage. Despite the rapid die-off of non-EB gram-negative bacteria in yogurt, screening for them using CVTA may be useful in heat-treated fermented dairy products with $\mathrm{pH}$ values higher than that of yogurt (e.g., cottage cheese). For example, Pseudomonas spp. are regularly linked to spoilage of cottage cheese through the formation of surface films and the production of degradative enzymes and metabolites (Cousin, 1982; Brocklehurst and Lund, 1985).

Although we hypothesize that the low $\mathrm{pH}$ of the yogurt was the primary factor contributing to the organism die-off reported in this study, other inhibitory factors inherent to Greek yogurt do exist. In particular, starter cultures may play a significant role in the die-off of postpasteurization bacterial contaminants. As part of the active fermentation process in yogurt, starter cultures grow to high levels and may outcompete other bacterial contaminants present in low numbers. In addition to utilizing the primary carbohydrate source (i.e., lactose) and decreasing the $\mathrm{pH}$ of the yogurt via organic acid production, starter cultures may also produce hydrogen peroxide early in product shelf life (Dave and Shah, 1997; Vedamuthu, 2007). Previous studies have found variations in both the $\mathrm{pH}$ and hydrogen peroxide content of yogurts made with various starter culture cocktails (Dave and Shah, 1997). Thus, the specific starter cultures used for fermentation may affect the postpasteurization survival of bacterial hygiene indicators present in yogurt. Similarly, intra-genus variability in bacterial hygiene indicator survival is also to be expected. However, for many of the genera evaluated here, we observed similar log differences between isolates in the same genus (Figure 1). Although future studies may allow further insight into the variability in die-off for strains in the same genus, the large data set presented here should be sufficient to allow for sciencebased decisions on selection of hygiene indicator tests for Greek yogurt. Even though we found good survival of most EB strains over $12 \mathrm{~h}$, previous reports of rapid die-off of many gram-negative hygiene indicators have prompted researchers to evaluate alternative organism groups capable of identifying yogurt postpasteurization contamination, namely yeasts and enterococci (Rohm et al., 1992; Birollo et al., 2001; Lourens-Hattingh and Viljoen, 2002). Although acid-tolerant yeasts and enterococci have been shown to survive in yogurt throughout product shelf life (Birollo et al., 2001; Lourens-Hattingh and Viljoen, 2002), traditional coliform detection methods are cheaper and faster than yeast and enterococci detection methods. Additionally, Birollo et al. (2001) concluded that coliforms are more frequent contaminants of dairy products than enterococci and may remain viable throughout fermentation and cold storage. For these reasons, Birollo et al. (2001) concluded that enterococci have little value as hygiene indicators in the industrial processes of yogurt. However, given that contamination of yogurts with acid-tolerant yeast via postpasteurization contamination or added nondairy ingredients represents the primary cause of premature spoilage in yogurt (Fleet, 1990), yeast testing typically would be valuable for yogurt processors, particularly when used in conjunction with testing for bacterial indicator organisms that detect a wider range of post-pasteurization contamination events.

\section{CVTA Detected the Highest Portion of Coliforms and EB Petrifilm Detected the Highest Portion of EB and Total Gram-Negative Bacteria in Inoculated Yogurt}

Among the 64 isolates, 4 (Aeromonas FSL C4-0005 and R5-0758; Brevundimonas FSL C4-0016 and C40057) did not show detectable growth on any of the 4 media and were not detected on the D-Count. Assessment of the different testing methods evaluated here was thus performed based on data from 60 different isolates. The 60 isolates in this data set included 42 coliforms. Each of the 5 methods evaluated yielded positive 
Table 1. Percent detection of hygiene indicator organism groups on crystal violet tetrazolium agar (CVTA), violet red bile glucose agar (VRBGA; Becton Dickinson, Sparks, MD), Enterobacteriaceae (EB) Petrifilm (3M, St. Paul, MN), the D-Count (bioMérieux, Marcy-l'Étoile, France), and Coliform Petrifilm (3M)

\begin{tabular}{lccc}
\hline Detection method & $\begin{array}{c}\text { Coliforms } \\
(\mathrm{n}=42)^{1}\end{array}$ & $\begin{array}{c}\text { Enterobacteriaceae } \\
(\mathrm{n}=54)^{2}\end{array}$ & $\begin{array}{c}\text { Gram-negative } \\
\text { bacteria }(\mathrm{n}=60)^{3}\end{array}$ \\
\hline CVTA & 98 & 83 & 80 \\
VRBGA & 90 & 91 & 82 \\
EB Petrifilm & 93 & 94 & 85 \\
D-Count & 90 & 93 & 83 \\
Coliform Petrifilm & 93 & 72 & 65 \\
\hline
\end{tabular}

${ }^{1}$ Forty-two isolates were classified as coliforms based on positive results when pure cultures were tested on Coliform Petrifilm (Hervert et al., 2016).

${ }^{2}$ Fifty-four isolates were classified as EB based on sequencing of partial $16 \mathrm{~S}$ rDNA.

${ }^{3}$ Of the total 64 gram-negative isolates tested, 60 isolates survived the 12-h hold in Greek yogurt and were detected using one or more testing methods. Isolates were classified as gram-negative organisms based on sequencing of partial 16S rDNA.

results (i.e., growth with typical colony characteristics for medium-based detection methods or $\geq 100$ counts/ $\mathrm{mL}$ for the D-Count) with $\geq 90 \%$ of the 42 coliform isolates inoculated into Greek yogurt (Table 1). Crystal violet tetrazolium agar yielded the highest percentage detection for coliforms (98\%; 41/42 isolates; Table 1). Among the 60 isolates, 54 represented EB; this includes all 42 coliform isolates. Crystal violet tetrazolium agar and Coliform Petrifilm yielded positive results with $83 \%(45 / 54)$ and $72 \%(39 / 54)$, respectively, of the EB isolates inoculated into Greek yogurt. By comparison, the EB-specific detection methods (i.e., EB Petrifilm, VRBGA, and D-Count) all detected $>90 \%$ of the EB isolates tested (Table 1). Among the total isolate set of 60 gram-negatives, EB Petrifilm showed the highest percentage detection $(85 \% ; 51 / 60)$. The remaining tests were less successful at detecting the 60 gram-negative isolates that survived the hold period with percentage detections ranging from $65 \%$ (Coliform Petrifilm) to $83 \%$ (D-Count; Table 1). Notably, CVTA only detected $80 \%(48 / 60)$ of the gram-negative isolates; the 12 isolates not detected with CVTA included 1 coliform, 8 noncoliform EB, and 3 non-EB gram-negative isolates.

\section{All Methods Evaluated Showed 100\% Specificity and Detected $\geq 80 \%$ of Their Respective Target Bacteria}

The sensitivity (true positive rate) and specificity (true negative rate) of each test was evaluated using data for 60 gram-negative isolates (which included 42 coliforms and $54 \mathrm{~EB}$ ), as detailed in the previous section (Table 2). Observed sensitivities of the detection methods ranged from 80 to $94 \%$ (Table 2). Coliform Petrifilm, the only coliform detection method evalu- ated, detected $93 \%$ (39/42) of the coliforms inoculated into Greek yogurt. All 3 EB detection methods exhibited comparable results with observed sensitivities of 91\% (49/54), 93\% (50/54), and 94\% (51/54) for VRBGA, D-Count, and EB Petrifilm, respectively (Table 2). Although EB Petrifilm and VRBGA require $24 \mathrm{~h}$ to obtain results, the D-Count delivers results in 13 $\mathrm{h}$, indicating the potential for shorter time-to-result with flow cytometry-based methods. Crystal violet tetrazolium agar, the only detection method for total gram-negative bacteria, had the lowest sensitivity of the 5 methods evaluated $(80 \%$; $48 / 60$; Table 2$)$. No false-positive test results were found for any of the detection methods evaluated, indicating $100 \%$ specificity with the isolates tested (Table 2), although we must note that relatively small numbers of nontarget bacteria were tested here. By comparison, a previous pure culture study of a larger isolate set found lower specificities for some methods (i.e., 89 and $92 \%$ for $\mathrm{EB}$ Petrifilm and VRBGA, respectively; Hervert et al., 2016). Throughout our study, we did not find evidence that the starter cultures in the inoculated products used had the ability to grow on selective media used (at least under the standard incubation times used here as described in the Materials and Methods). This was demonstrated through the absence of any growth or detection when uninoculated, negative-control yogurt samples were tested using selective media and the D-Count, respectively. However, further evaluation of detection methods for exclusivity (i.e., absence of detection of gram-positive bacteria and starter cultures found in a given product) may be valuable.

Overall, 4 methods exhibited reduced sensitivities for target bacteria inoculated into Greek yogurt compared with those expected based on pure culture testing 
Table 2. Expected and observed sensitivities and specificities ${ }^{1}$ for crystal violet tetrazolium agar (CVTA), violet red bile glucose agar (VRBGA; Becton Dickinson, Sparks, MD), Enterobacteriaceae (EB) Petrifilm (3M, St. Paul, MN), the D-Count (bioMérieux, Marcy-l'Étoile, France), and Coliform Petrifilm (3M)

\begin{tabular}{lccccc}
\hline & \multicolumn{2}{c}{ Sensitivity $(\%)$} & & \multicolumn{2}{c}{ Specificity (\%) } \\
\cline { 2 - 3 } \cline { 5 - 6 } Detection method & Observed & Expected & & Observed & Expected \\
\hline CVTA & $80^{2}$ & $87^{2}$ & & $\mathrm{NA}^{3}$ & $\mathrm{NA}$ \\
VRBGA & $91^{4}$ & $93^{4}$ & & $100^{5}$ & $100^{5}$ \\
EB Petrifilm & $94^{4}$ & $100^{4}$ & & $100^{5}$ & $100^{5}$ \\
D-Count & $93^{4}$ & $89^{4}$ & & $100^{5}$ & $100^{5}$ \\
Coliform Petrifilm & $93^{6}$ & $100^{6}$ & & $100^{7}$ & $100^{7}$ \\
\hline
\end{tabular}

${ }^{1}$ Sensitivity (true positive rate) was defined as the proportion of true positives that are correctly identified as such; specificity (true negative rate) was defined as the proportion of true negatives that are correctly identified as such; observed results are based on the inoculated yogurt tests reported here; expected results for inoculated yogurt tests were based on pure culture results reported by Hervert et al. (2016).

${ }^{2}$ Calculations for CVTA were based on 60 isolates that were detected with one or more of the testing methods used here.

${ }^{3} \mathrm{NA}=$ not applicable.

${ }^{4}$ Calculations for EB methods were based on $54 \mathrm{~EB}$ isolates tested.

${ }^{5}$ Calcuations for the EB methods were based on 6 non-EB isolates tested.

${ }^{6}$ Calculations for coliform method were based on 42 coliform isolates tested.

${ }^{7}$ Calculations for the coliform method were based on 18 noncoliform isolates.

for the same isolates (Hervert et al., 2016). Crystal violet tetrazolium agar and Coliform Petrifilm showed the largest difference between expected and observed sensitivities with decreases of 7 percentage points, respectively (Table 2). Discrepancies between expected and observed sensitivities represented 16 instances of unexpected negative test results across each of the 5 detection methods (Supplemental Table S1; https:// doi.org/10.3168/jds.2016-11553). Unexpected negative results represented either isolates that yielded positive results when tested in pure culture but did not exhibit growth or detection when tested in Greek yogurt (11 isolates; Supplemental Table S1), or isolates that yielded positive results with typical colony characteristics when tested in pure culture but atypical colony characteristics yielding negative results when tested in Greek yogurt (5 isolates; Supplemental Table S1). The 11 instances of unexpected absence of growth occurred on CVTA (2/11), Coliform Petrifilm (3/11), EB Petrifilm $(4 / 11)$, VRBGA (1/11), and the D-Count (1/11). The 5 instances of unexpected atypical colony characteristics included 3 Salmonella isolates (FSL A5-0214, A50218, and A5-0285), 1 Yersinia isolate (FSL L6-0049), and 1 Rahnella isolate (FSL P4-0879) that exhibited colorless colonies rather than red colonies on CVTA. Reduced recovery of injured cells following exposure to stress conditions is a well-documented occurrence that is commonly encountered with selective and differential media types (Ray, 1986; Smith et al., 2013). We hypothesize that the lack of recovery or typical colony characteristics on selective and differential media may be attributed to cell injury induced from the low $\mathrm{pH}$ environment of the yogurt.

Surprisingly, 3 isolates (Yersinia FSL R5-0761 and R5-0600; Plesiomonas FSL Y1-0254) showed positive test results on the D-Count when tested from Greek yogurt, even though they tested negative on the D-Count in pure culture (Hervert et al., 2016); these instances led to the D-Count method showing a higher observed sensitivity for the testing of isolates inoculated into Greek yogurt compared with the expected sensitivity based on pure culture testing (Table 2). We hypothesize that these isolates may have benefited from the 1-g addition of yogurt to the enrichment broth that allowed them to grow to detectable levels when tested on the D-Count. Additionally, 1 Acinetobacter isolate (FSL C4-0087) grew with atypical colony characteristics on CVTA when tested in pure culture but grew with typical red colonies when tested in Greek yogurt.

\section{CONCLUSIONS}

Our data indicated that gram-negative bacteria showed a range of survival capabilities over a 12-h incubation in Greek yogurt. Although the presence of any gram-negative bacteria in commercially produced yogurt indicates postpasteurization contamination (or, much less likely, pasteurization failure), our data indicated that EB testing methods allow for sensitive detection that yields positive results with the genera most likely encountered in low-pH fermented dairy products. The fact that Pseudomonas spp. did not 
present positive results with EB detection methods represents a concern for higher $\mathrm{pH}$ dairy products (e.g., fresh cheeses and fluid milk), where Pseudomonas spp. are a considerable spoilage concern. However, the use of methods that do not detect Pseudomonas is less of a concern in yogurt because Pseudomonas seem to show rapid die-off and are not linked to spoilage issues. The continued development of methods to detect hygiene indicator organisms that target EB and are affordable, rapid, and reliable thus continues to be of great importance to dairy processors. Although our data identified reliable EB tests, we also showed that novel methods (e.g., flow cytometry-based technologies) had potential for more rapid detection that reduces the 24- to 48-h turnaround times typical of traditional methods; importantly, time savings associated with rapid detection methods will allow for reduced product hold times until release. Overall, our data suggested that EB testing provided an appropriate approach for monitoring the hygienic status of yogurt.

\section{ACKNOWLEDGMENTS}

This publication was made possible by an unrestricted gift from Chobani Inc. (Norwich, NY), supporting innovative research in dairy quality. We thank them for their generous contribution and continued support.

\section{REFERENCES}

Aykut, S. A., and Z. Y. Oezbas. 1994. Growth of Yersinia enterocolitica and Aeromonas hydrophila in acidophilus yoghurt. Aust. J. Dairy Technol. 49:90-92.

Bachrouri, M., E. J. Quinto, and M. T. Mora. 2002. Survival of Escherichia Coli O157:H7 during storage of yogurt at different temperatures. J. Food Sci. 67:1899-1903.

Birollo, G. A., J. A. Reinheimer, and C. G. Vinderola. 2001. Enterococci vs non-lactic acid microflora as hygiene indicators for sweetened yoghurt. Food Microbiol. 18:597-604.

Brocklehurst, T. F., and B. M. Lund. 1985. Microbiological changes in cottage cheese varieties during storage at $+7^{\circ} \mathrm{C}$. Food Microbiol. $2: 207-233$.

Canganella, F., M. L. Nespica, D. Giontella, and L. D. Trovatelli. 1999. Survival of Enterobacter cloacae and Pseudomonas paucimobilis in yoghurts manufactured from cow's milk and soymilk during storage at different temperatures. Microbiol. Res. 154:15-21.

Champagne, C. P., R. R. Laing, D. Roy, A. A. Mafu, M. W. Griffiths, and C. White. 1994. Psychrotrophs in dairy products: Their effects and their control. Crit. Rev. Food Sci. Nutr. 34:1-30.

Costa Dias, M. A., A. S. Sant'Ana, A. G. Cruz, J. A. F. Faria, C. A. Fernandes de Oliveira, and E. Bona. 2012. On the implementation of good manufacturing practices in a small processing unity of mozzarella cheese in Brazil. Food Contr. 24:199-205.

Cousin, M. A. 1982. Presence and activity of psychrotrophic microorganisms in milk and dairy products: A review. J. Food Prot. 45:172-207.

Cusato, S., A. H. Gameiro, C. H. Corassin, A. S. Sant'Ana, A. G. Cruz, J. A. F. Faria, and C. A. F. de Oliveira. 2013. Food safety systems in a small dairy factory: Implementation, major challeng- es, and assessment of systems' performances. Foodborne Pathog. Dis. 10:6-12.

Dave, R. I., and N. P. Shah. 1997. Viability of yoghurt and probiotic bacteria in yoghurts made from commercial starter cultures. Int. Dairy J. 7:31-41.

Davidson, P. M., L. A. Roth, and S. A. Gambrel-Lenarz. 2004. Coliform and other indicator bacteria. Pages 187-226 in Standard Methods for the Examination of Dairy Products. 17th ed. M. Wehr, ed. Am. Public Health Assoc., Washington, DC.

Desai, N. T., L. Shepard, and M. A. Drake. 2013. Sensory properties and drivers of liking for Greek yogurts. J. Dairy Sci. 96:7454-7466.

Feng, P., S. D. Weagant, M. A. Grant, and W. Burkhardt. 2002. Enumeration of Escherichia coli and the coliform bacteria. Chapter 4 in Bacteriological Analytical Manual. 8th ed. US Food and Drug Administration, Center for Food Safety and Applied Nutrition, College Park, MD. Accessed Feb. 16, 2016. http://www.fda.gov/ Food/FoodScienceResearch/LaboratoryMethods/ucm064948.htm.

Fleet, G. H. 1990. Yeasts in dairy products. J. Appl. Bacteriol. 68:199 211

Frank, J. F., and A. E. Yousef. 2004. Tests for groups of microorganisms. Pages 227-248 in Standard Methods for the Examination of Dairy Products. 17th ed. M. Wehr, ed. Am. Public Health Assoc., Washington, DC.

Goel, M. C., D. C. Kulshrestha, E. H. Marth, D. W. Francis, J. G. Bradshaw, and R. B. Read. 1971. Fate of coliforms in yogurt, buttermilk, sour cream, and cottage cheese during refrigerated storage. J. Milk Food Technol. 34:54-58.

Hervert, C. J., A. S. Alles, N. H. Martin, K. J. Boor, and M. Wiedmann. 2016. Evaluation of different methods to detect microbial hygiene indicators relevant in the dairy industry. J. Dairy Sci. 99:7033-7042.

Huck, J. R., N. H. Woodcock, R. D. Ralyea, and K. J. Boor. 2007. Molecular subtyping and characterization of psychrotolerant endospore-forming bacteria in two New York State fluid milk processing systems. J. Food Prot. 70:2354-2364.

Imhoff, J. 2005. Enterobacteriales. Pages 587-850 in Bergey's Manual of Systematic Bacteriology. D. Brenner, N. Krieg, J. Staley, G. Garrity, D. Boone, P. De Vos, M. Goodfellow, F. Rainey, and K. H. Schleifer, ed. Springer, New York, NY.

Lourens-Hattingh, A., and B. C. Viljoen. 2002. Survival of dairyassociated yeasts in yoghurt and yoghurt-related products. Food Microbiol. 19:597-604.

Martin, N. H., N. R. Carey, S. C. Murphy, M. Wiedmann, and K. J. Boor. 2012. A decade of improvement: New York State fluid milk quality. J. Dairy Sci. 95:7384-7390.

Masiello, S. N., N. H. Martin, A. Trmčić, M. Wiedmann, and K. J. Boor. 2016. Identification and characterization of psychrotolerant coliform bacteria isolated from pasteurized fluid milk. J. Dairy Sci. 99:130-140.

Nassib, T. A., M. Z. El-Din, and W. M. El-Sharoud. 2006. Effect of thermophilic lactic acid bacteria on the viability of Salmonella serovar Typhimurium PT8 during milk fermentation and preparation of buffalo's yogurt. Int. J. Dairy Technol. 59:29-34.

Ranieri, M. L., and K. J. Boor. 2009. Short communication: Bacterial ecology of high-temperature, short-time pasteurized milk processed in the United States. J. Dairy Sci. 92:4833-4840.

Ray, B. 1986. Impact of bacterial injury and repair in food microbiology: Its past, present and future. J. Food Prot. 49:651-670.

Rohm, H., F. Eliskases-Lechner, and M. Bräuer. 1992. Diversity of yeasts in selected dairy products. J. Appl. Bacteriol. 72:370-376.

Schröder, M. J. A. 1984. Origins and levels of post pasteurization contamination of milk in the dairy and their effects on keeping quality. J. Dairy Res. 51:59-67.

Shaker, R. R., T. M. Osaili, and M. Ayyash. 2008. Effect of thermophilic lactic acid bacteria on the fate of Enterobacter sakazakii during processing and storage of plain yogurt. J. Food Saf. $28: 170-182$.

Silva, R., A. G. Cruz, J. A. Faria, M. M. Moura, L. M. Carvalho, E. H. Water, and A. S. Sant'Ana. 2010. Pasteurized milk: Efficiency of 
pasteurization and its microbiological conditions in Brazil. Foodborne Pathog. Dis. 7:217-219.

Smith, A. R., A. L. Ellison, A. L. Robinson, M. Drake, S. A. McDowell, J. K. Mitchell, P. D. Gerard, R. A. Heckler, and J. L. McKillip. 2013. Enumeration of sublethally injured Escherichia coli O157:H7 ATCC 43895 and Escherichia coli strain B-41560 using selective agar overlays versus commercial methods. J. Food Prot. 76:674-679.

Tacket, C. O., J. P. Narain, R. Sattin, J. P. Lofgren, C. Konigsberg, R. C. Rendtorff, A. Rausa, B. R. Davis, and M. L. Cohen. 1984. A multistate outbreak of infections caused by Yersinia enterocolitica transmitted by pasteurized milk. JAMA 251:483-486.

Trmčić, A., K. Chauhan, D. J. Kent, R. D. Ralyea, N. H. Martin, K. J. Boor, and M. Wiedmann. 2016. Coliform detection in cheese is associated with specific cheese characteristics, but no association was found with pathogen detection. J. Dairy Sci. 99:6105-6120.

US FDA (Food and Drug Administration). 2011. Standards for grade "A" milk and milk products. Pages 27-30 in Grade "A" Pasteur- ized Milk Ordinance. Standards for grade "A" milk and milk products. US Department of Health and Human Services, Public Health Service, Washington, DC.

US Treasury Department. 1914. Bacterial standard for drinking water. Pages 2959-2966 in Public Health Report. Vol. 29. US Treasury Department, Washington, DC.

USDA-ERS (Economic Research Service). 2015. Dairy products: Per capita consumption, United States (Annual). Accessed Aug. 15 2016. http://www.ers.usda.gov/data-products/dairy-data.aspx.

Vangay, P., E. B. Fugett, Q. Sun, and M. Wiedmann. 2013. Food Microbe Tracker: A web-based tool for storage and comparison of food-associated microbes. J. Food Prot. 76:283-294.

Vedamuthu, E. R. 2007. Starter cultures for yogurt and fermented milks. Pages 89-116 in Manufacturing Yogurt and Fermented Milks. R. C. Chandan, ed. Blackwell Publishing Ltd., Ames, IA.

Wolfe, B. E., J. E. Button, M. Santarelli, and R. J. Dutton. 2014 Cheese rind communities provide tractable systems for in situ and in vitro studies of microbial diversity. Cell 158:422-433. 